\title{
Preface to the Special Issue Unconventional Computing 2008
}

\author{
Cristian S. Calude · José Felix Costa
}

Published online: 21 October 2010

(C) Springer Science+Business Media B.V. 2010

\section{Introduction}

This special issue contains five articles selected from the papers presented at the 7 th International Conference on Unconventional Computing, UC 2008, held in Vienna, Austria on 25-28 August 2008. "Unconventional Computing", http://www.cs.auckland.ac.nz/ research/groups/CDMTCS/conferences/uc/uc.html, is the primary international conference dedicated to all aspects of unconventional computing, theory as well as experiments and applications.

Giorgio Delzanno and Laurent Van Begin study computational properties of extended P systems that model creation, dissolution, fusion, and cloning of membranes in their paper "On the verification of membrane systems with dynamic structure".

The paper "Quantum security in wireless sensor networks", by Naya Nagy, Marius Nagy and Selim Akl, proposes a new security solution based on quantum cryptography.

Turlough Neary proves, in the paper "On the computational complexity of spiking neural P systems", that no spiking neural P system (with a constant number of neurons) is capable of simulating Turing machines with less than exponential time and space overheads. He also constructs a universal extended spiking neural $\mathrm{P}$ system with exhaustive use of rules and 10 neurons that simulates Turing machines in linear time.

In the paper "Output concepts for accelerated Turing machines" Petrus Potgieter and Elemér Rosinger study the problem of rigorously defining the output of the infinite computation performed by an accelerated Turing machine.

Linmin Yang, Yong Wang and Zhe Dang study a new computation paradigm, inspired by $\mathrm{P}$ systems, defined over a multiset of communicating objects in their paper "Automata and processes of multisets of communicating objects". They prove that the Presburger

C. S. Calude (西)

University of Auckland, Auckland, New Zealand

e-mail: cristian@cs.auckland.ac.nz

J. F. Costa

IST Technical University of Lisbon, Lisbon, Portugal 
reachability problem is decidable for single-process service automata, but undecidable in the multiprocess case.

We thank all reviewers of the papers, A. Abbott, A. Alhazov, M. Gheorghe, G. Mauri, V. Mitrana, A. Păun, M. Perez-Jimenez, L. Staiger, and C. Zandron, for their comments and suggestions, an important contribution to this special issue. 